\title{
DESCRIBING THE COMPLETE EFFECTS OF PROGRAMS
}

\author{
Ria Follett \\ Department of Computer Science \\ University of New South Wales \\ ABSTRACT
}

\begin{abstract}
It is important to consider the total effect of program in order to produce correct software. If the effects of the program are formally considered, it is usually done by describing the invariants and predicate transformers of program segments. Instead of using predicate transformers, a more powerful concept of a passback pair is introduced. Passback pairs allow the effects of a program to be described to a level suitable for the problem at hand. Methods of automatically deriving passback pairs of various degrees of refinement are discussed. These methods are then applied in an automatic program synthesis system, PROSYN.
\end{abstract}

\section{INTRODUCTION}

The complete effects of programs must be considered in any system which either uses the program or attempts to prove facts about the program. The complete effects of a program can be characterized by the way any relation (such as a relation on the values of variables) is altered by the execution of the program. Knowledge of the effects of any given program segment, is especially vital to any program verification system, and to any automatic programming system, as 'intuition' cannot be used. These effects must be described formally, so they can be used mechanically, or derived automatically. A method of describing the complete effects of a program is discussed here, together with methods of analysing programs to discover these effects. This analysis may be done either automatically or manually. These descriptions can then be used in program verification or program synthesis. An automatic program synthesis system which uses these descriptions of the effects of a program, and analyses programs for their effects, is discussed.

\section{PRECONDITIONS}

Most program verifiers, (King 1969, and Deutsch 1973), and some automatic program synthesis systems, (Waldinger 1975) use predicate transformers to describe the effects of a program segment. The predicate transformers calculate the preconditions of any given relation. The preconditions are those relations whose truth guarantees that a given relation is valid after the associated program segment is executed. Thus to achieve (or verify) the relation $R$ after the program segment, it is sufficient to achieve (or verify) 
its preconditions before the segment.

The precondition is calculated using a predicate transformer. The predicate transformer is usually given as a rule or function on the relation $R$. For example, for the program segment

$$
v:=a
$$

the relation $R$ is true after the segment if its precondition is true before the segment. The precondition is the relation obtained by substituting a for $\mathrm{v}$ in $\mathrm{R}$.

The predicate transformer describes the effects of a program segment. If the precondition of $R$ is the same as $R$, then the program does not affect $R$. If the relations are not identical, then the predicate transformer gives the complete effect of the segment on the relation $R$.

The main problem with this method is that it is extremely difficult to generate the predicate transformers of complicated program segments such as loops. The study of predicate transformers for loops is usually limited to finding invariants of the loop. An invariant is a relation whose precondition is identical to itself, thus unaltered by the predicate transformer. (Manna 1974, Dijkstra 1976)

However in order to synthesise programs automatically, it is not sufficient to find only the invariants of loops. The preconditions of the loop for other specific relations are required so that those relations may be achieved and verified. This means that predicate transformers of program segments must be obtained to a varying degree of generality.

In program synthesis, it is fortunately not usually necessary to find predicate transformers applicable to all relations. For example, it may be sufficient for the above assignment statement to consider only the predicate transformers for $R$ where $R$ does not contain $v$. The precondition will then be $R$ itself. In other circumstances, the preconditions for other relations are required. To allow the predicate transformers to be generated at the appropriate level of generality for the relations to wich it may be applied, the concept of predicate transformers is generalised to the passback pair.

\section{THE PASSBACK PAIR}

A passback pair is an ordered pair $(D, E)$ of a predicate $D$, and a function $f$, on an arbitrary relation $R$. It is a description of the effects of a particular program segment $p$ on a relation $R$. The second element of the ordered pair, $F$, denotes the function which calculates the precondition of $R$. The first element, $D$, is the domain of relations on which $f$ is defined. If $D(R)$ is satisfiable, then $f$ can modify $R$, thus giving the precondition of $R$. If $D(R)$ is not satisfiable, then the precondition of $R$ cannot be calculated using $f$. There is a range of passback pairs valid for any program segment. The passback pair (FALSE, I) is valid for all program seg- 
ments, where I is the identity function, meaning that no relation will be passed back over this segment to produce a precondition. are

Two possible passback pairs for the assignment statement $v:=a$

( $v$ is not contained in $R$, I )

and (TRUE, substitute a for $v$ in $R$ ).

The first means that only relations $R$, where $R$ does not contain the variable ' $v$ ', may be passed back. These relations will be passed back unaffected by the assignment statement.

The second means that all relations $R$ may be passed back by replacing all the occurrences of ' $\mathrm{v}$ ' in $R$. by ' $a$ '.

The process of obtaining a more precise passback pair is called refining the passback pair. Refining the passback pair implies enlarging the domain, with a corresponding modification to $f$. It is the refining process which gives the passback pair more power than the usual concept of predicate transformers. For many, especially complex, program segments, it is difficult to obtain a complete predicate transformer. For these segments, a passback pair with a limited domain may be easily derived. If this description is sufficient for the problem at hand, no further work need be done. Howev$e r$, if the description is not sufficiently precise, the passback pair may be refined (either by an automatic system, or manually) to increase the size of the domain. The amount of work thus required for program verification (or synthesis) is reduced without affecting the validity of the resulting program.

\section{DERTVING PASSBACK PAIRS}

The passback pairs for primitive program segments (called PRIMITIVES) must be supplied initially by the user. The primitives required depend on the particular programming domain, but may be limited, say, to the assignment statement. The passback pair for any other program segment can be derived only by combining the passback pairs of basic program segments. This can often be done automatically.

The control structures which will be analysed consist of straight line programs, the branch statement (IF - THEN - ELSE) and the insertion of recursive procedure calls. The automatic programming system discussed in Section 5 is also limited to these constructs. The programs are then analysed in stages. First, a passback pair is found for the program segment. If it is sufficient for the current purpose, then it is used. Otherwise the passback pair is refined to produce a more accurate description.

In the following examples, the two program segments P1 and P2 are described by the passback pairs $(D 1, f 1)$ and $(D 2, f 2)$ respectiveIy. These program segments may be primitives, or segments which have al ready been analysed. 


\subsection{Combining Straight Line Programs}

The program segment formed by processing the first primitive before the second, denoted by P1;P2 may be described by the passback pair (D2 A D1.f2, E1.f2). The passback pair description means that, for a goal $R$ to be passed back using the function f1.f2, it must be possible to satisfy both $\mathrm{D} 2(\mathrm{R})$ and $\mathrm{D} 1 . \mathrm{f} 2(\mathrm{R})$ simultaneously.

\subsection{Branches}

Branches are in the form IF A THEN P1 ELSE P2. The following description assumes that the test $A$ has no side effect and can be computed as often as desired. The passback pair for the branch may be described as

$$
\begin{aligned}
& (D 1 \wedge D 2 \wedge(f 1(R)=f 2(R)), f 1(R)) \\
& \text { or }(D 1 \wedge D 2, f 1(R) \wedge \notin 2(R)) \\
& \text { or (D1^D2, IF A THEN } f 1(R) \text { ELSE } f 2(R)) \text {. }
\end{aligned}
$$

The first passback pair is obtained by allowing only the relations which satisfy both $\mathrm{D} 1$ and $\mathrm{D2}$, and are altered in an identical manner along both branches, to be passed back over the branch.

The second passback patr is a refined version of the first and is obtained by passing the relation back over both paths. The program needs to achieve both $f(R)$ and $f 2(R)$, to ensure that the relation $R$ is true after the branch.

However, the relation $f(R)$ is required only if $A$ is true, and otherwise $\mathrm{f} 2(\mathrm{R})$ is required. This means that the second passback function may be simplified, giving the complete passback function of IF A THEN $\mathrm{EL}(\mathrm{R})$ ELSE $\mathrm{f} 2(\mathrm{R})$.

Note that the value of $A$ cannot be used in the domain on which the passback function is defined as $A$ may have been modified by $P l$ or $\mathrm{P} 2$. Thus $(\mathrm{A} \wedge \mathrm{D} 1) \mathrm{OR}(\sim \mathrm{A} \wedge \mathrm{D} 2)$ is not a valid domain.

For example - consider a program segment that sorts two numbers $x$ and $y$. The program segment generated may be

IF $x \in y$ THEN NIL

$$
\begin{aligned}
\text { ELSE BEGIN temp }:=x ; \\
z:=y ; \\
y:=\text { temp } \\
\text { END }
\end{aligned}
$$

Let this new subprogram be called $\operatorname{SoRT} 2(\mathrm{x}, \mathrm{y})$. As the test $\mathrm{x} \leqslant \mathrm{y}$ has no side effect the above discussion applies. A possible passback pair is (temp $\notin R \wedge x \notin R \wedge y \notin R, I)$, where $\notin$ denotes "is not contained in". This passback pair is derived from using the passback pair of " $v:=\exp ^{\prime \prime}$ as $(v \notin R, I)$. This passback pair allows only relations in which neither temp nor $x$ nor $y$ occurs, to be passed back. Although valid, it is unnecessarily restrictive in its domain. It does not allow relations such as $x<z$ AND $y<z$ to be passed back even though this relation is in fact unaltered by $\operatorname{SORT2}(x, y)$.

If a refined version of the passback pair for "v:=exp" such as (TRUE, substitute exp for $v$ in $R$ ) is used, then the else branch can 
be described (using Section 4.1 for combining straight line programs) as (TRUE, $R^{\prime \prime \prime)}$

where $R^{\prime \prime}$ is obtained by substituting $x$ for temp in $R^{\prime \prime}$

and $R^{\prime \prime}$ by substituting $y$ for $x$ in $R^{\prime}$

and $R^{\prime}$ by substituting temp for $y$ in $R$.

The passback pair for the THEN section is (TRUE, I). These may be combined giving

$\left(\mathrm{R}^{\prime \prime} \equiv \mathrm{R}, \mathrm{I}\right)$.

This passback pair means that whenever $x$ and $y$ can be interchanged in the relation without altering the relation, the relation $c$ an be passed back unaffected. If this description is not sufficient for the problem at hand, the passback pair may be further refined giving the passback pair

(TRUE , IF $x \leqslant y$ THEN $R$ ELSE $R^{\prime \prime \prime) ~}$

where $R^{\prime \prime}$ is defined as above.

\subsection{Recursive Procedures}

Recursive procedures cannot easily be analysed for the associated predicate transformer. This is because the number of procedure calls is usually unknown a priori. It is here that the passback pair simplifies the description. For any recursive procedure a non-trivial passback pair can be obtained in the following manner.

Firstly, consider the procedure obtained by deleting all the recursive procedure calls from the procedure. A passback pair can be constructed for this procedure with an identity passback function. For example, the passback pair could have as its domain the relations which do not contain any variables that may be set by the procedure. This passback pair can then be generalised over all the recursive procedure calls, to give a valid passback pair for the recursive procedure.

For example, consider the procedure MAX, which places the maximum element of an array in the zeroth element of that array:

MAX $<=$ IF $n<1$ THEN NIL

$$
\begin{gathered}
\text { ELSE REGIN } \operatorname{SORT2}(x(n), x(n)) ; \\
\text { END }
\end{gathered}
$$

A passback pair for the procedure obtained by deleting the recursive procedure call, $\operatorname{MAX}(n-1)$, is

$$
(x(0) \notin R \wedge x(n) \notin R, I) \text {. }
$$

Generalised over the recursive procedure calls, a passback pair for $\operatorname{MAX}(n)$ is

$$
\text { ( FORALL } i=0, n \times(i) \notin R, x) \text {. }
$$

\subsection{Refining the Passback Pair}

The passback pair defined above corresponds to a loop invariant. It is clearly not the most precise passback pair. In many cases this passback pair suffices in program synthesis or verification but there are a number of refinements that may be applied. 


\subsubsection{The Domain of the Passback Pair may be Widened by Using Invariants}

A loop invariant is a relation among the program variables which is unaltered, at a given point in the 10op, by executing the 1oop. A relation which is an invariant both at the start and end of the loop may enlarge the domain of a passback pair.

Given any such invariant $N$, it is possible to refine the passback pair $(D, I)$ to $(0 \vee N, I)$ which increases the size of the domain over which $f$ is defined. Some methods of automatically generating invariants are given by Elspas(1974), Wegbreit (1974) and Katz and Manna(1976).

\subsubsection{Passback Functions may be Derived}

The above discussion leads to passback pairs for recursive procedures of the form $(D, I)$ where I is the identity function. Invariants can be used to increase the size of the domain D, but still lead to an identity passback function. However, it is usually possible to derive a passback function where the segments also have a non-identity passback function. From section 4.1 it is possible to combine passback functions as

$\mathrm{fl}$. f2.f $3 . \cdots$.f $\mathrm{n}$

The problem is to find a general function (possibly in terms of commutative operators FORALL and EXISTS) describing this sequence.

In the previous example, MAX, a possible passback pair for $\operatorname{SORT} 2(x, y)$ is

( TRUE , $\mathrm{R}$ AND ( $\mathrm{R}$ with $\mathrm{y}$ and $\mathrm{x}$ interchanged)).

For the case where $i=n=1$, the relation $R$ may be passed back over MAX resulting in the relation R1, where $R 1$ is

$R 1$ : $R$ AND ( $R$ with $\times(1) \times(0)$ interchanged).

This means that the relation $\mathrm{R} 1$ must be achieved before $\operatorname{SORT} 2(x(0), x(1))$ in order to achieve $R$ after the SORT2 procedure call.

For the case $n=2$ the relation which needs to be achieved is

$\mathrm{R} 2$ : R1 AND (R1 with $\mathrm{x}(2) \mathrm{x}(0)$ interchanged).

This may be general ised as

$R$ AND FORALL $k \leqslant n$ FORALL $j \leqslant k$ ( $R$ with $x(k) x(j)$ interchanged). The passback pair for the loop then becomes

( TRUE, R AND FORALL $k \leqslant n$ FORALL $j \leqslant k$ (R with $x(k) \times(j)$

interchanged)).

Stated plainly, this means that $R$ is valid after $\operatorname{MAX}(n)$ if all relations obtained by the various permutations of interchanging $x(k)$ and $x(j)$ are valid before $\operatorname{MAX}(n)$. 


\section{ISING PASSBACK PAIRS IN PROGRAM SYNTHESIS}

The concept of passback pairs has been applied to program synthesis. The implementation, called PROSYN, takes a list of goals and achieves these in turn. Goals may be passed back through previously achieved program segments as appropriate. This effectively reorders the initial list of goals. Once achieved, goals are protected. Passback pairs are also used to ensure that the insertion of program steps does not interfere with protected goals. Recursive procedures may be synthesised, and passback pairs are derived and refined where appropriate.

The passback pairs have been very useful in program synthesis, as they ensure that the program produced is valid. As the synthesiser automatically derives the passback pairs from simple primitives, a simple passback pair can be derived and used as required. Usually quite simple passback pairs resulted in quite complex programs. This meant that only a simple analysis of the program segments was required to produce valid programs. Some automatic refining methods were used if the simple analysis failed.

This approach has succeeded in synthesising a wide variety of programs. Various domains such as BLOCKS, LISTS and numerous NUMBER domains have been used. In the NUMBR domain (augmented to include arrays) the programs synthesised include

- SORT2

- MAX

- SUM of an array

- solving two simultaneous equations

- REMAINDER from dividing two numbers

- moving elements up and down arrays

- sorting arrays

- adding, multiplying and transposing matrices

- inverting matrices

- finding eigenvectors of matrices

- finding the zeros of a function

- solving a set of linear simultaneous equations.

Note that, in all the above cases, a program to achieve the result was obtained.

\section{EXAMPLE}

As a simple example to illustrate the use of passback pairs in automatic program synthesis, consider the problem of shifting elements down an array. In this example, unless the elements are shifted in the correct sequence, the side effects of the program will alter the values of the array itself. The problem may be expressed by the goal

FORALL $i=2, n \quad v(i-1)=s(i)$

where $v$ is the array and $s$ is the initial value of that array.

This example is one of many synthesised by the automatic program synthesiser PROSYN. Both the synthesis of the program and the 
analysis of the relevant effects occur automatically. The following is an informal description of the steps which took place in the synthesis.

PROSYN may have the array $v$ predefined with the initial values $s$, or this may be supplied by the user. The user then requests PROSYN to synthesise a program to achieve the goal. The goal is currently specified in prefix notation, being

(NEWGOAL "(FORALL (i 2 n) (EQUAL $(v(i-1))$ (s i)))).

PROSYN then synthesises the subroutine to do this, together with the main program calling this subroutine. A complete description of the subroutine is al so produced, allowing that subroutine to be used in other contexts. For ease of understanding, the general subroutine name generated by PROSYN for the subroutine has been altered to "SHIFT".

Given this goal, PROSYN attempts induction, as FORALL is defined in the current domain to operate on a set of values, in this case with $i$ ranging from 2 to $n$. The problem is split into two subproblems,

$$
v(n-1)=s(n) \text { AND FORALI } i=2, n-1 \quad v(i-1)=s(i) \text {. }
$$

The problem can be split into these two subproblems only if $n>2$. This condition must first be synthesised, giving the program segment with the set of values $(2, n)$ generalised to $(m, n)$ as :

$\operatorname{SHIFT}(m, n)<-$ IF $n<m$ THEN NIL

$$
\text { ELSE ... }
$$

PROSYN then attempts the split goals in turn. The first goal $v(n-1)=s(n)$ can be achieved using the assignment primitive

$$
\text { VAR: }=\text { EXP. }
$$

As $s(n)$ is a value instead of a variable, PROSYN must find a variable with that value. As no variable has yet been altered, it finds that initial1y $s(n)=v(n)$. This results in the primitive

$v(n-1):=v(n)$.

As no other goal has yet been achieved the primitive can be simply inserted without interfering with any goal, giving the prom gram segment:

$$
\begin{aligned}
& \operatorname{SHIFT}(\mathrm{m}, \mathrm{n})<- \text { IF } \mathrm{n}<\mathrm{m} \text { THEN NIL } \\
& \qquad \begin{aligned}
\operatorname{ELSE} 0 \mathrm{BEGIN} \\
50 \mathrm{~V}(\mathrm{n}-1):=\mathrm{v}(\mathrm{n}) \\
100 \mathrm{END}
\end{aligned}
\end{aligned}
$$

The relations that have been developed, either to achieve the goal, or used in achieving the goal are then protected. These are

$s(n)=v(n) \quad$ is protected from step 0 to step 50

$v(n-1)=s(n)$ is protected from step 50 to step 100.

The goal remaining to be accomplished at step 100 is

FORALL $i=m, n-1 \vee(i-1)=s(i)$.

This is an instance of the original goal, so a recursive call is attempted. Firstiy termination is established. Then the effect of the function is calculated in the form of a passback pair. Using 
the methods in Section 4.3 a passback pair of $\operatorname{SHIFT}(m, n-1)$ is calculated to be (FORALL $i=m-1, n-2 v(i) \notin R, I$ ).

As this does not interfere with the protected relation $v(n-1)=s(n)$, it may be inserted. However the precondition of the function $\operatorname{SHIFT}(m, n-1)$ is calculated to be

FORALL $i=m, n-1 \quad v(i)=s(i)$.

This precondition cannot be achieved as $v(n-1)=s(n)$ is protected. This causes the insertion of the recursive function call to fail.

The failure resulted from the order in which the goals were attempted. Induction was first attempted on $\mathrm{n}$ rather than on $\mathrm{m}$. If induction on $m$ were attempted first the procedure would have resulted in success. This ordering was chosen on purpose to illustrate the use of passback pairs in the program synthesis.

PROSYN then attempts to pass back the goal

FORALL $i=m, n-1 \quad v(i-1)=s(i)$

over the program step $v(n-1):=v(n)$. The goal may be passed over this by replacing $v(n-1)$ by $v(n)$ in that goal, but as $v(n-1)$ is not in the goal, the goal will be passed back unaffected. This results in the goal to be achieved before 50 as

FORALL $i=m, n-1 \quad v(i-1)=s(i)$.

Once again a recursive function call may be inserted. The preconditions are true by the initial conditions, so the resulting program is:



with the protected goals:-

$\begin{array}{clrr}\text { FORALL } i=m, n-1 & v(i)=s(i) & \text { from } 0 & \text { to } 25 \\ \text { FORALL } i=m, n-1 & v(i-1)=s(i) & \text { from } 25 \text { to } 100 \\ v(n)=s(n) & \text { from } 0 \text { to } 50 \\ v(n-1)=s(n) & \text { from } 50 \text { to } 100 .\end{array}$

The initial program written simply consists of a subroutine cal1 $\operatorname{SHIFT}(2, n)$.

The description of the function generated was

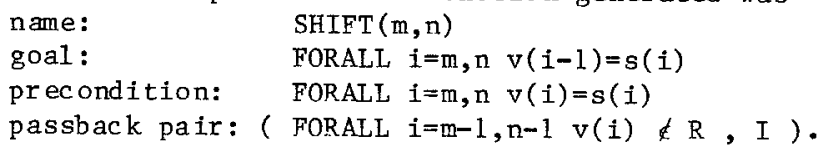

The above subroutine was synthesised with only a simple description of the passback pair relating to that function. Most subroutines that were synthesised required only a simple description such as the one derived here. In some cases, the simple description was not adequate. Only these cases need a more completed analysis of the overall effects, thus saving in effort for the other cases. However, any passback pair derived is sufficient to verify the completed program. 


\section{CONCLUSION}

Using the passback pair has given more flexibility in the description of programs than the use of simple predicate transformers. The correctness of a program in the sense that a particular goal is achieved can be proved using passback pairs without the need to discover the details of all the possible effects. This has been particularly useful in automatic program synthesis, where only the effects of a program on given relations need to be derived, and where complicated recursive procedures then need to be analysed, if possible automatically. For recursive procedures predicate transformers cannot be easily derived. The process of refining a passback pair means that valid programs can be synthesised with a minimum of analysis for the complete effects of the program. Further analysis of its effects occurs only if this is required for the problem currently being attempted. This has resulted in the automatic synthesis and analysis of a wide variety of programs.

The problem of deriving predicate transformers, considered in the context of refining passback pairs, is still very difficult. Some strategies for refining passback pairs exist, but there is no general algorithm for arbitrarily complicated programs, in the same way that there is no general algorithm for discovering invariants and predicate transformers. However, the generalisation of predicate transformers to passback pairs means that complete analysis is not usually required to synthesise the required program.

\section{REFERENCES}

Deutsch L.P. (1973) 'An Interactive Program Verifier', PhD Thesis, Dept of Computer Sci., University of California, Berkeley.

Dijkstra F.W. (1976) 'A Discipline of Programming', Prentice-Hal1, N.J.

Elspas B. (1974) 'The Semiautomatic Generation of Inductive Assertions for Proving Program Correctness', Research Rep, Stanford Research Institute, Menlo Park, Calif.

Katz S. and Manna 2. (1976) 'Logical Analysis of Programs', Comm ACM Vol 19 No 4.

King J. (1969) 'A Program Verifier', PhD Thesis, Dept of Computer Science, Carnegie-Mellon Uni., Pittsburgh, Pa.

Manna Z. (1974) 'Mathematical Theory of Computation', McGraw-Hil1 Book Co.

Waldinger R. (1975) 'Achieving Several Goals Simultaneously', Stanford Research Institute A.I. Center Technical Note 107.

Wegbreit B (1974) 'The Synthesis of Loop Predicates', Comm ACM 17,2 p 102-112. 